\title{
The Shannon Entropy-Based Node Placement for Enrichment and Simplification of Meshes
}

\author{
Vladimir Savchenko ${ }^{1}$, Maria Savchenko ${ }^{2}$, Olga Egorova ${ }^{3}$, and Ichiro Hagiwara ${ }^{3}$ \\ ${ }^{1}$ Hosei University, Tokyo, Japan \\ vsavchen@k.hosei.ac.jp \\ ${ }^{2}$ InterLocus Inc.Tokyo, Japan \\ savchenko.m.aa@m.titech.ac.jp \\ 3 Tokyo Institute of Technology, Tokyo, Japan \\ egorova.o.aa@m.titech.ac.jp, hagiwara@mech.titech.ac.jp
}

\begin{abstract}
In this paper, we present a novel simple method based on the idea of exploiting the Shannon entropy as a measure of the interinfluence relationships between neighboring nodes of a mesh to optimize node locations. The method can be used in a pre-processing stage for subsequent studies such as finite element analysis by providing better input parameters for these processes. Experimental results are included to demonstrate the functionality of our method.
\end{abstract}

Keywords: Mesh enrichment, Shannon entropy, node placement.

\section{Introduction}

Construction of a geometric mesh from a given surface triangulation has been discussed in many papers (see [1] and references therein). Known approaches are guaranteed to pass through the original sample points that are important in computer aided design (CAD). However, results of triangulations drastically depend on uniformity and density of the sampled points as it can be seen in Fig.1. Surface remeshing has become very important today for CAD and computer graphics (CG) applications. Complex and detailed models can be generated by $3 \mathrm{D}$ scanners, and such models have found a wide range of applications in $\mathrm{CG}$ and CAD, particularly in reverse engineering. Surface remeshing is also very important for technologies related to engineering applications such as finite element analysis (FEA). Various automatic mesh generation tools are widely used for FEA. However, all of these tools may create distorted or ill-shaped elements, which can lead to inaccurate and unstable approximation. Thus, improvement of the mesh quality is an almost obligatory step for preprocessing of mesh data in FEA. Recently, sampled point clouds have received much attention in the CG community for visualization purposes (see [2], [3]) and CAD applications (see [4], [5], [6]). A noise-resistant algorithm [6] for reconstructing a watertight surface from point cloud data presented by Kolluri et al. ignores undersampled regions; nevertheless, it seems to us that some examples show that undersampled areas need an improvement by surface retouching or enrichment algorithms. In some 
applications, it is useful to have various, for instance, simpler versions of original complex models according to the requirements of the applications, especially, in FEA. In addition to the deterioration in the accuracy of calculations, speed may be sacrificed in some applications. Simplification of a geometric mesh considered here involves constructing a mesh element which is optimized to improve the elements shape quality using an aspect ratio (AR) as a measure of the quality.

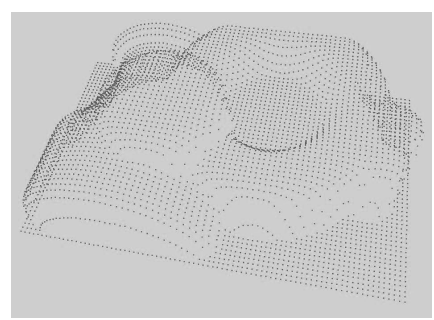

(a)

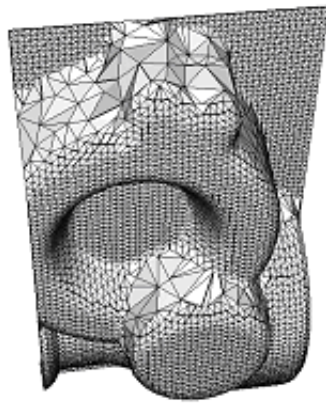

(b)

Fig. 1. Surface reconstruction of a "technical data set". (a) Cloud of points (4100 scattered points are used). (b)Triangulation produced by Delaunay-based method (N triangular elements: 7991, N points: 4100).

In this paper, we present an attempt of enrichment of mesh vertices according to AR-based entropy which is the analog of the Shannon entropy [7]. Further it is called A-entropy. We progressively adapt the new coming points by performing elementary interpolation operations proposed by Shepard [8] (see also [9] for more references) for generating the new point instances until an important function $I_{f}$ (in our case, a scalar which specifies the ideal sampling density) matches some user-specified values. To optimize node coordinates during simplification process (edge collapsing), A-entropy is also implemented.

Recently, a wide scope of papers addressed the problem of remeshing of triangulated surface meshes, see, for instance, [10], [11] and references therein where surface remeshing based on surface parameterization and subsequent lifting of height data were applied. However, the main assumption used is that geometric details are captured accurately in the given model. Nevertheless, as it can be seen from the Darmstadt benchmark model (technical data set shown in Fig. 1), a laser scanner often performs non-uniform samples that leads to under-sampling or the mesh may have holes corresponding to deficiencies in point data. In theory, the problem of surface completion does not have a solution when the plane of the desirable triangulation is not planar; and, especially, begins to go wrong when the plane of triangulation is orthogonal to features in the hole boundary (so called, crenellations features). See a good discussion of the problem in [12]. Let us emphasize that our approach is different from methods related to reconstruction of surfaces from scattered data by interpolation methods based, for instance, on minimum-energy properties (see, for example, [13]). In our case, an 
approximation of the original surface (triangular mesh) is given. In some applications, it is important to preserve the initial mesh topology. Thus, our goal is to insert new points in domains where the $I_{f}$ function does not satisfy the user-specified value. The main contribution of the paper is a novel algorithm of a vertex placement which is discussed in details in Section 2.

\section{Vertex Placement Algorithm}

The approach is extremely simple and, in theory, is user dependent. In an analogy to a hole filling, the user defines an area, where enrichment may be done, that is, the user selects a processing area with no crenellations. In practice, all object surface can be considered as the processing area (as it has been done in the example shown in Fig. 1).

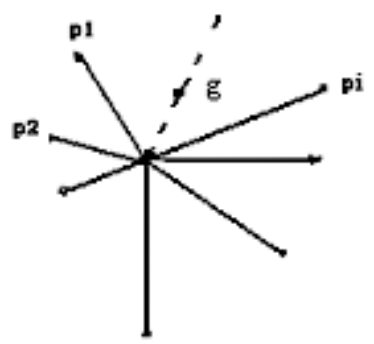

Fig. 2. Scheme of a new point generation. $p_{1}, p_{2}, p_{i}$ are points of the neighborhood. The dashed line is a bisector of the "empty" sector, $g$ is the generated point.

After that the algorithm works as follows:

1. Define a radius of sampling $R_{s}$ as an analog of the point density; for instance, for the "technical data set" the radius equal to 1 . It can be done automatically by calculating average point density.

2. For each point of the user-defined domain, select $K$ nearest points $p$ that are in $R_{s}$ neighborhood. If $K$ is less (or equal) than the user-predefined number of the neighborhood points (in our experiments - 6) and the maximum angle of the "empty" area is larger (or equal) the user-predefined angle (in our experiments $-90^{\circ}$ ), generate a new point $g$ with the initial guess provided by a bisector of the "empty" sector as shown in Fig. 2.

3. Select a new neighborhood of the point $g$; it can be slightly different from the initial set of points. This is done in the tangent plane (projective plane) defined by neighborhood points.

4. Perform a local Delaunay triangulation.

5. Find points forming a set of triangles with the common node $g$ (a star) as shown in Fig. 3(a). Calculate the new placement of the center of the star $g$ using technique described below (Fig. 3(b)). 
6. And on the lifting stage, calculate the local $z$-coordinates of $g$ by Shepard interpolation. In our implementation, we use the compactly supported radial basis function [14] as the weight function.

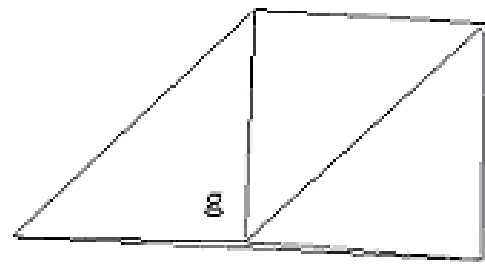

(a)

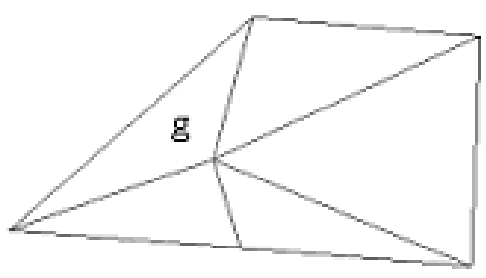

(b)

Fig. 3. (a) An initial star. (b) The final star.

The key idea of the fifth step is to progressively adapt the newly created points throw a few iterations. That is, an area with low sampling density will be filled in accordance with points generated on the previous steps. In order to obtain a "good" set of the new (approximated) points coordinates, we need a measure of a "goodness" of triangulations arising from randomly coming points. It is natural to use a mesh quality parameter, AR of the elements of a star, for such a measure. In the case of a triangular mesh, AR can be defined as a ratio of the maximum edge length to the minimum edge length of an element. Nevertheless, according to our experiments it is much better to use an information $M_{i}\left(M_{i}\right.$ is the AR of the $i$-th triangle of the star) associated with respect to a point $g$ (Fig. 3) of the star in an analogy with the Shannon entropy [8], which defines the uncertainty of a random variable, and can be a natural measure for the criterion used in the enrichment algorithm. Shannon defined the entropy of an ensemble of messages: if there are $N$ possible messages that can be sent in one package, and message $m$ is being transmmited with probability $p_{m}$, then the entropy is as follows

$$
S=-\sum_{1}^{N} p_{m} \log \left(p_{m}\right) .
$$

Intuitively, we can use AR-based entropy, with respect to the point $g$ as follows

$$
S=-\sum_{i=0}^{N} M_{i} / M_{t} \log \left(M_{i} / M_{t}\right),
$$

where $M_{t}$ is the summarized AR value of a star, $N$ is the number of faces of the star. From the statistical point of view, a strict definition of the Shannon entropy for a mesh, which we denoted as A-entropy and used in our algorithm, is provided as follows: consider discrete random variable $\xi$ with distribution: 


$$
\left(\begin{array}{llll}
x_{1} & x_{2} & \ldots & x_{n} \\
p_{1} & p_{2} & \ldots & p_{n}
\end{array}\right)(\star)
$$

where probabilities $p_{i}=\mathrm{P}\left\{\xi=x_{i}\right\}$. Then divide an interval $0 \leq x<1$ into such intervals $\Delta_{i}$ that the length of $\Delta_{i}$ equals to $p_{i}$. Random variable $\xi$ is defined as $\xi=x_{i}$, when $\gamma \in \Delta_{i}$ has distribution $(\star)$. Suppose we have a set of empirically received numbers $\gamma_{1}=a_{1}, \ldots, \gamma_{n}=a_{n}$ written in its increasing order, where $a_{i}$ is the AR of the $i$-th element of the neighborhood with the point $g$ as a center. Let these numbers define a division of an interval $a_{1} \leq x<a_{n}$ into $\Delta_{i}=a_{i}$ - $a_{i-1}$. In our case, the parameter $a_{i}$ has its minimal value equal to 1 , which is not necessarily achieved in given sampling data. Constructing the one-to-one correspondence between $1 \leq x<a_{n}$ and $0 \leq x<1$, the following probabilities can be written:

$$
p_{1}=\frac{a_{1}-1}{a_{n}-1}, p_{2}=\frac{a_{2}-a_{1}}{a_{n}-1}, p_{n}=\frac{a_{n}-a_{n-1}}{a_{n}-1} \ldots
$$

Thus, we can define the random variable with the distribution as follows

$$
\left(\begin{array}{llll}
a_{1} & a_{2} & \ldots & a_{n} \\
p_{1} & p_{2} & \ldots & p_{n}
\end{array}\right)
$$

Its probability values are used in formula (3) for A-entropy:

$$
A=-\sum_{1}^{N} p_{i} \log \left(p_{i}\right), p_{i}=\frac{a_{i}-a_{i-1}}{a_{n}-1}, p_{0}=1 .
$$

The value $\mathrm{A}$ of A-entropy depends on the coordinates of the center of the star (point $g$ in Fig. 3). Thus, the problem of maximization of the value $\mathrm{A}$ is reduced to the problem of finding the new coordinates of this center (Fig. 3(b)) and is considered as the optimization problem. For solving this optimization problem, we use the downhill simplex method of Nelder and Mead [15].

\section{Experimental Results}

In practice, as it can be seen in Fig. 4, implementation of the algorithm discussed above leads to a reasonable surface reconstruction of areas with initially low sampling density (see Fig. 1). The number of scattered points in the initial model is 4100, after enrichment the number of points was increased up to 12114. For decreasing the number of points we simplify this model and the final number of points is 5261 .

Our triangular mesh simplification method uses predictor-corrector steps for predicting candidates for edge collapsing according to a bending energy [16] with the consequent correction of the point placement in simplified mesh. Let us notice that the main idea of our simplification approach is to provide minimal local surface deformation during an edge collapse operation. 


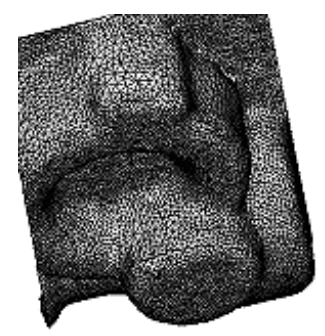

(a)

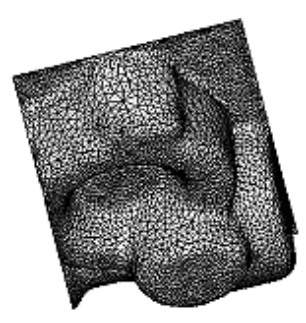

(b)

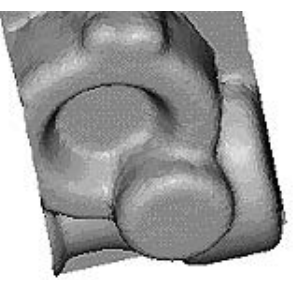

(c)

Fig. 4. The mechanical data set. (a) Mesh after enrichment. (b) Mesh after simplification. (c) Shaded image of the final surface.

At each iteration step:

- candidate points for an edge collapse are defined according to a local decimation cost of points belonging to a shaped polygon.

- after all candidates have been selected, we produce a contraction of the edge with choosing an optimal vertex position by using A-entropy according to the fifth step of the algorithm (see Section 2).

To detail the features of the proposed point placement scheme, Fig. 5 presents results of applying well known or even classical surface simplification algorithms (tools can be found in [17]) and our method. We show fragment of a "Horse" model (the initial AR value is equal to 1.74; here and further, the average value of the AR is used) after the mesh simplification produced by the different simplification techniques.

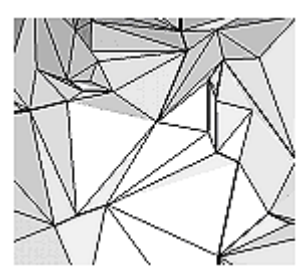

(a)

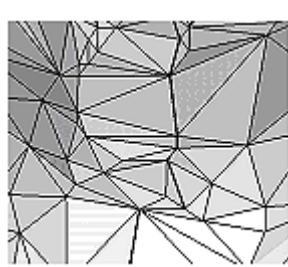

(b)

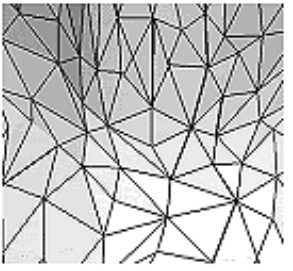

(c)

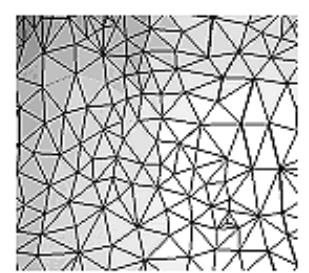

(d)

Fig. 5. Mesh fragments of the "Horse" model after simplification ( $\sim 13 \%$ of original elements) by using: (a) Progressive simplification method; (b) Method based on a global error bound; (c) Method based on a quadric error metric; (d) Our method

The volume difference between the initial model and simplified one by our technique is $0.8 \%$; the final $\mathrm{AR}$ value is equal to 1.5 . The global error bound method demonstrates the worst results; the volume difference is $1.3 \%$, the final $\mathrm{AR}$ value is equal to 2.25. At a glance of the model visualization and the volume preservation, the best method, without any doubt, is the method based on the quadric error metric, see [18]. However, there is a tradeoff between attaining a 
high quality surface reconstruction and minimization of AR. As a result, the final $\mathrm{AR}$ value is equal to 2 and many elongated and skinny triangles can be observed in the mesh.

\section{Concluding Remarks}

In this paper, we introduce the notion of AR-based entropy (A-entropy) which is the analog of the Snannon entropy. We consider the enrichment technique and the technique for improving the mesh quality which are based on this notion. The mesh quality improvement in presented simplification technique can be compared with smoothing methods based on the averaging of the coordinates, such as Laplacian [19] or an angle based method of Zhou and Shimada [20]. These methods have an intrinsic drawback such as a possibility of creating inverted triangles. In some non-convex domains, nodes can be pulled outside the boundary. Implementation of the entropy-based placement in simplification algorithm decreases a possibility that a predicted point does not create an inverted triangle, but does not guarantee that such event does not occur at all. However, producing operations in the tangent plane allows sufficiently easy avoiding creation of inverted triangles. Interpolation based on the Shepard method produces excessive bumps. In fact, it is a well known feature of the original Shepard method. More sophisticated local interpolation schemes such as [21] and others can be implemented to control the quality of interpolation. Matters related to feature preserving shape interpolation have to be considered in the future. We have mentioned that it might be natural to use AR (the mesh quality parameter) of the elements of a star as a measure for providing a reasonable vertex placement. Nevertheless, we would like to emphasize that according to our experiments, in many cases it does not lead at all to a well-founded estimate of a point $g$. It might be a rational approach to use the Shannon entropy as a measure of the inter-influence relationships between neighboring nodes of a star to calculate optimal positions of vertices. We can see in Fig. 5 that shapes of mesh elements after implementation of our method differ significantly from results of applying other simplification methods. Meshes in Fig. 5(a, b, c) are more suitable for visualization than for numerical calculations. Improvement of meshes of a very low initial quality, for instance, the "Horse" model simplified by the global error bound method, takes many iteration steps to attain $\mathrm{AR}$ value close to our result and after implementation of Laplacian smoothing to the model shown in Fig. 5(b) the shape of the model is strongly deformed. After implementation of Laplacian smoothing (300 iteration steps) to the "Horse" model, simplified by the quadric error metric method, AR and volume difference between the original model and improved one become 1.6 and 5.2\%, correspondingly. Examples demonstrated above show that the mesh after applying our method is closer to a computational mesh and can be used for FEA in any field of study dealing with isotropic meshes. 


\section{References}

1. Frey P. J.: About Surface Remeshing. Proc.of the 9th Int.Mesh Roundtable (2000) 123-136

2. Alexa, M., Behr, J.,Cohen-Or, D., Fleishman, S., Levin, D., Silvia, C. T.: Point Set Surfaces. Proc. of IEEE Visualization 2001 (2002) 21-23

3. Pauly, M., Gross, M., Kobbelt, L.: Efficient Simplification of Point-Sampled Surfaces. Proc. of IEEE Visualization 2002(2002) 163-170

4. Hoppe, H., DeRose, T., Duchamp, T., McDonald, J.,Stuetzle, W.: Surface Reconstruction from Unorganized Points. Proceedings of SIGGRAPH 92 (1992) 71-78

5. Amenta, N., Choi, S., Kolluri, R.: The Powercrust. Proc. of the 6th ACM Symposium on Solid Modeling and Applications (1980) 609-633

6. Kolluri, R., Shewchuk, J.R., O’Brien, J.F.: Spectral Surface Reconstruction From Noisy Point Clouds. Symposium on Geometry Processing (2004) 11-21

7. Blahut, R.E.: Principles and Practice of Information Theory. Addison-Wisley (1987)

8. Shepard, D.: A Two-Dimensional Interpolation Function for Irregularly Spaced Data. Proc. of the 23th Nat. Conf. of the ACM (1968) 517-523

9. Franke, R., Nielson, G.,: Smooth Interpolation of Large Sets of Scattered Data. Journal of Numerical Methods in Engineering 15 (1980) 1691-1704

10. Alliez, P., de Verdiere, E.C., Devillers, O., Isenburg, M.: Isotropic Surface Remeshing. Proc.of Shape Modeling International (2003)49-58

11. Alliez, P., Cohen-Steiner, D., Devillers, O., Levy, B., Desburn, M.: Anisotropic Polygonal Remeshing. Inria Preprint 4808 (2003)

12. Liepa, P.: Filling Holes in Meshes. Proc. of 2003 Eurographics/ACM SIGGRAPH symp.on Geometry processing 43 200-205

13. Carr, J.C., Mitchell, T.J., Beatson, R.K., Cherrie, J.B., Fright, W.R., McCallumn, B.C., Evans, T.R.: Filling Holes in Meshes. Proc.of SIGGRAPH01 (2001) 67-76

14. Wendland, H.: Piecewise Polynomial, Positive Defined and Compactly Supported Radial Functions of Minimal Degree. AICM 4 (1995) 389-396

15. Nelder, J.A., Mead, R.: A simplex Method for Function Minimization. Computer J. 7 (1965) 308-313

16. Bookstein, F.L.: Morphometric Tools for Landmarks Data. Cambridge University Press (1991) Computer J. 7 (1965) 308-313

17. Schroeder, W., Martin, K., Lorensen,B.: The Visualization Toolkit. Ed.2 Prentice Hall Inc. (1998)

18. Garland, M.: A Multiresolution Modeling: Survey and Future Opportunities. Proc. of EUROGRAPHICS, State of the Art Reports (1999)

19. Bossen, F.J., Heckbert, P.S.: A Pliant Method for Anisotropic Mesh Generation. Proc. of the 5th International Meshing Roundtable (1996) 63-74

20. Zhou, T., Shimada, K.: An Angle-Based Approach to Two-dimensional Mesh Smoothing. Proc.of the 9th International Meshing Roundtable (2000) 373-384

21. Krysl, P., Belytchko, T.: An Efficient Linear-precision Partition of Unity Basis for Unstructured Meshless Methods. Communications in Numerical Methods in Engineering 16 (2000) 239-255 